Randomised controlled trial

\section{Epidural steroid injections are not effective for patients with lumbar spinal stenosis}

\author{
10.1136/ebmed-2014-110083
}

\section{Steven J Atlas}

Department of Medicine, Massachusetts General Hospital and Harvard Medical School, Boston, Massachusetts, USA

Correspondence to: Dr Steven Atlas, Massachusetts General Hospital, Department of Medicine, 50 Staniford Street, Room 966. Boston, MA 02114, USA; satlas@mgh.harvard.edu

Commentary on: Friedly JL, Comstock BA, Turner JA, et al. A randomized trial of epidural glucocorticoid injections for spinal stenosis. N Engl J Med 2014;371:11-21.

\section{Context}

Degenerative lumbar spinal stenosis is a common cause of low back and leg pain in older individuals. The evidence supporting non-surgical treatments, including oral medications, physical treatments and spinal injections, is limited. ${ }^{1}$ Despite the lack of strong evidence supporting the use of epidural steroid injections (ESIs) for lumbar spinal stenosis, their use has increased dramatically since $2000 .^{2}$ This multicentre, randomised trial compared the effectiveness of ESIs with lidocaine to epidural injections of lidocaine alone.

\section{Methods}

Patients were at least 50-years-old with pain and functional impairment attributable to lumbar spinal stenosis of the central canal, with no prior lumbar surgery or ESI within the past 6 months. Four hundred patients were randomly assigned to either a standard ESI (glucocorticoids) with lidocaine, or an injection of lidocaine alone, with potential for a repeat study injection at 3 weeks. Twenty-six anaesthesiologists, physiatrists and radiologists experienced in epidural injections from 16 US sites performed the procedures. The primary outcomes assessed at 6 weeks were an average pain rating for buttock, hip or leg pain in the past week and functional impairment measured by the Roland-Morris Disability Questionnaire (RMDQ). Patients, treating physicians and research staff performing baseline and outcome assessments were blinded to treatment assignment.

\section{Findings}

Baseline patient characteristics were similar among groups, and they had the same 6-week follow-up rate of 96.5\%. Clinically and statistically significant decreases in pain and RMDQ scores were seen in both groups. There were no significant differences between groups in the average pain (adjusted difference -0.2 points, 95\% CI -0.8 to 0.4 ) or RMDQ scores (adjusted difference -1.0 points; $95 \% \mathrm{CI}-2.1$ to 0.1 ). A prespecified subgroup analysis of interlaminar $(\mathrm{n}=282)$ and transforaminal injection revealed a small but statistically significant benefit to RMDQ score for interlaminar injection in the ESI with lidocaine group (adjusted difference -1.4 points; $95 \%$ CI -2.8 to $-0.1, \mathrm{p}<0.05$ ), but no benefit to average pain. There was no difference in primary outcomes for the subgroup who received transforaminal injections. Among secondary outcomes, most showed no group differences at 6 weeks, but there were small, statistically significant improvements in pain and RMDQ in the ESI with lidocaine group at 3 weeks. Patients who received an ESI with lidocaine were more satisfied with their treatment than those who received lidocaine alone (67\% vs 54\%, $\mathrm{p}=0.01$ ).

\section{Commentary}

This study adds to a growing body of research demonstrating the lack of important benefit of ESIs in patients with lumbar spinal stenosis. Overall, these results raise serious concerns about the overuse of ESIs in patients with symptomatic central canal stenosis. Although this study showed a small, statistically significant effect favouring the ESI group at 3 weeks, this benefit was no longer apparent at 6 weeks. Moreover, this statistical difference among treatment groups at 3 weeks would not be considered a clinically meaningful effect. Other secondary pain and functional status outcomes also showed no significant benefit at 6 weeks.

Should this study change current practice? Patients who remain symptomatic and functionally impaired despite initial empiric therapy with oral medications, physical treatments and time, should be engaged by providers to explore options and risks/benefits of various treatments with a shared decision-making and educational approach. ${ }^{3}$ Given the findings of short-term benefit, one could argue this could include ESIs. ${ }^{4}$ As an example, a highly symptomatic patient unable to actively participate in physical therapy due to pain and functional impairment could be considered for a single ESI as a way to engage her/him in exercise-based treatments. However, the lack of longer term benefit and the potential risks of ESIs suggest that using a series of injections to manage patients over time cannot be endorsed in the absence of more compelling evidence. ${ }^{4}$

This study leaves several unanswered questions. By focusing on individuals with central canal stenosis, it does not address patients undergoing injections for primarily foraminal and lateral recess stenosis. The subgroup analysis suggesting greater effectiveness for interlaminar techniques requires further investigation. Finally, one may question whether the lidocaine only control group represents a true placebo arm. Although it is hard to understand the physiological basis for a short-acting anaesthetic providing prolonged benefit, the current results argue for increased use of saline controls. Regardless of these limitations, the current study provides strong evidence that ESIs for patients with symptoms due to central canal lumbar spinal stenosis do not offer prolonged benefit compared to lidocaine only epidural injections.

Competing interests SA receives salary support through Massachusetts General Hospital as a medical editor for the Informed Medical Decisions Foundation, which is now a part of Healthwise, a not-for-profit foundation that develops and distributes patient education and decision support materials.

Provenance and peer review Commissioned; internally peer reviewed.

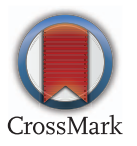

\section{References}

1. Ammendolia C, Stuber KJ, Rok E, et al. Nonoperative treatment for lumbar spinal stenosis with neurogenic claudication. Cochrane Database Syst Rev 2013;8:CD010712.

2. Manchikanti L, Pampati V, Falco FJ, et al. Assessment of the growth of epidural injections in the medicare population from 2000 to 2011. Pain Physician 2013;16: E349-64.

3. Chou R, Loeser JD, Owens DK, et al. Interventional therapies, surgery, and interdisciplinary rehabilitation for low back pain: an evidence-based clinical practice guideline from the American Pain Society. Spine 2009;34:1066-77.

4. Kreiner DS, Shaffer WO, Baisden JL, et al. North American Spine Society. An evidence-based clinical guideline for the diagnosis and treatment of degenerative lumbar spinal stenosis (update). Spine J 2013;13:734-43. 